\title{
Room-Temperature Magnetism of Ceria Nanocubes by Inductively Transferring Electrons to Ce Atoms from Nearby Oxygen Vacancy
}

\author{
Yue Kang ${ }^{1,2} \cdot$ Qiang Leng $^{1}$ - Donglin Guo ${ }^{1}$ Dezhi Yang ${ }^{1}$ Yanping $\mathrm{Pu}^{2} \cdot$ Chenguo $\mathrm{Hu}^{1}$
}

Received: 2 May 2015/Accepted: 1 July 2015/Published online: 19 August 2015

(C) The Author(s) 2015. This article is published with open access at Springerlink.com

\begin{abstract}
Ceria $\left(\mathrm{CeO}_{2}\right)$ nanocubes were synthesized by a hydrothermal method and weak ferromagnetism was observed in room temperature. After ultraviolet irradiation, the saturation magnetization was significantly enhanced from $\sim 3.18 \times 10^{-3}$ to $\sim 1.89 \times 10^{-2} \mathrm{emu} \mathrm{g}^{-1}$. This is due to the increase of oxygen vacancies in $\mathrm{CeO}_{2}$ structure which was confirmed by X-ray photoelectron spectra. The first-principle calculation with Vienna ab-initio simulation package was used to illustrate the enhanced ferromagnetism mechanism after calculating the density of states (DOSs) and partial density of states (PDOSs) of $\mathrm{CeO}_{2}$ without and with different oxygen vacancies. It was found that the increase of oxygen vacancies will enlarge the PDOSs of Ce $4 \mathrm{f}$ orbital and DOSs. Two electrons in one oxygen vacancy are respectively excited to $4 \mathrm{f}$ orbital of two $\mathrm{Ce}$ atoms neighboring the vacancy, making these electron spin directions on $4 \mathrm{f}$ orbitals of these two Ce atoms parallel. This superexchange interaction leads to the formation of ferromagnetism in $\mathrm{CeO}_{2}$ at room temperature. Our work indicates that ultraviolet irradiation is an effective method to enhance the magnetism of $\mathrm{CeO}_{2}$ nanocube, and the firstprinciple calculation can understand well the enhanced magnetism.
\end{abstract}

Keywords UV irradiation $\cdot$ Oxygen vacancies $\cdot$ Saturation magnetism $\cdot$ Spin direction $\cdot$ Superexchange interaction

\section{Introduction}

Nanocrystalline ceria $\left(\mathrm{CeO}_{2}\right)$ has attracted much attention due to its fascinating mechanical and physic-chemical properties, as well as wide applications in various fields, including polishing materials [1, 2], automobile exhaust catalysts [3-5], fuel cell materials [6, 7], gas sensors [8, 9], high temperature superconducting materials [10], ultraviolet ray detectors, etc. [11, 12]. Recently, Zhang et al. [13] reported that $\mathrm{CeO}_{2}$ nanoparticles have great potential for

Dezhi Yang

dzyang@cqu.edu.cn

$\triangle$ Chenguo $\mathrm{Hu}$

hucg@cqu.edu.cn

1 Department of Applied Physics, Chongqing University, Chongqing 400044, People's Republic of China

2 School of Public Affairs, Chongqing University, Chongqing 400044, People's Republic of China multifunctional therapeutic applications in cancer therapy. Flytzani-Stephanopoulos et al. [14] used different nanostructured $\mathrm{CeO}_{2}$ materials as a support to deposit gold clusters and found that $\mathrm{Au} / \mathrm{CeO}_{2}$ catalysts showed a strong support shape effect in the water-gas shift reaction. Most of the properties of cerium oxide was found to be related with oxygen vacancies in the structure [15-18].

It is well known that $\mathrm{CeO}_{2}$ with perfect structure is paramagnetic. When one of oxygen atoms is removed, two electrons will be left and localized strongly at the f-level traps on two Ce atoms. This will cause the formal valence of two neighboring $\mathrm{Ce}$ atoms changing from +4 to +3 , and therefore resulting in the formation of magnetism. Lee and co-workers [17] examined the localization behavior of $\mathrm{CeO}_{2}$ with various degrees of oxygen deficiency as well as the associated magnetic properties and their origins using firstprinciples methods in the Vienna ab-initio simulation package (VASP). Choudhury and Choudhury [10] investigated how oxygen vacancies and cationic $\mathrm{Ce}^{3+}$ defects in ceria affect the band gap property by changing its structural 
regularity and then control its visible luminescence. In addition, vacancies in some other non-magnetic materials can also induce magnetism. Si et al. [19] found that vacancies in hexagonal boron nitride nanosheets (h-BN) could generate magnetic moments and form ferromagnetism, and they conducted some experiments to test their discovery.

Although some methods could generate magnetism change such as spin-reorientation transition (SRT) in ultrathin $\mathrm{Ni}$ films grown on $\mathrm{Cu}$ (100) [20], few papers report the experimental methods of obtaining oxygen vacancies and changing the valence state. Recently, Qin et al. [21] improved the magnetic properties of semiconductors and found that UV irradiation could significantly enhance the magnetism of the (111) twinned $\mathrm{BaTiO}_{3}$ crystallites. They demonstrated that magnetism might originate from the increase in oxygen vacancies and $\mathrm{Ti}^{3+}$ cations formed by capturing an excited electron from $\mathrm{Ti}^{4+}$ under UV irradiation. Enlightened by this report, we speculate that the valence state might also change (from $\mathrm{Ce}^{4+}$ to $\mathrm{Ce}^{3+}$ ) under UV irradiation. Herein, the optical and magnetic properties of $\mathrm{CeO}_{2}$ under UV irradiation were investigated and the electron density of states (DOSs) and the electron partial density of states (PDOSs) were calculated via density functional theory for a better understanding of the phenomenon.

\section{Experimental Details}

\subsection{Synthesis of $\mathrm{CeO}_{2}$ Nanocubes}

The uniform organic-capping-free $\mathrm{CeO}_{2}$ nanocubes were synthesized using a hydrothermal process [22]. Briefly, $434.3 \mathrm{mg}$ of $\mathrm{Ce}\left(\mathrm{NO}_{3}\right)_{3} \cdot 6 \mathrm{H}_{2} \mathrm{O}$ and $1.6 \mathrm{mg}$ of $\mathrm{Na}_{3} \mathrm{PO}_{4}$ were mixed in $40 \mathrm{~mL}$ deionized water and sonicated for $30 \mathrm{~min}$. The mixed solution was then transferred into a Teflon-lined autoclave with $50 \mathrm{~mL}$ capacity. The vessel was sealed and heated at $200{ }^{\circ} \mathrm{C}$ for $24 \mathrm{~h}$ in an electric furnace. Then the product was collected and cleansed by centrifugation at $10,000 \mathrm{r} \mathrm{min}^{-1}$ for $5 \mathrm{~min}$ and re-dispersed twice in ethanol. The precipitate was then calcined at $400{ }^{\circ} \mathrm{C}$ for $4 \mathrm{~h}$ and collected for further characterization.

\subsection{UV Irradiation on $\mathrm{CeO}_{2}$}

The dried sample was uniformly distributed in a square crucible and put in a darkroom to irradiate for $24 \mathrm{~h}$ under the UV lamp.

\subsection{Characterization of $\mathrm{CeO}_{2}$}

The powder X-ray diffraction (XRD) patterns were recorded on PANalytical Empyrean equipped with a PIXcel with a $1 \mathrm{D}$ detector at $40 \mathrm{kV}$ and $40 \mathrm{~mA}$. Field emission scanning electron microscopy (FESEM) was carried out with a Nova 400 Nano microscope to analyze the morphology and size of the product. Transmission electron microscopy (TEM) was used to analyze the morphology and size of the samples with JEOL-400EX. The X-ray photoelectron spectra (XPS) were conducted by an ESCALab MKI X-ray photoelectron spectrometer, using non-monochromatized $\mathrm{Mg} \mathrm{K} \alpha \mathrm{X}$-ray as the excitation source. The reflection spectrum of the sample was measured using UV-3600 (SHIMADZU) UV-VIS-NIR Spectrophotometer. Magnetism measurement was carried out on superconducting quantum interference device (SQUIDs) measurement.

\subsection{Computation of Magnetism}

First-principles methods, implemented in the Vienna abinitio simulation package (VASP) [23], were used to study the magnetism of $\mathrm{CeO}_{2}$ without and with oxygen vacancies. Because of the strong Coulomb interaction of the localized $\mathrm{Ce} 4 \mathrm{f}$ electrons, the standard density functional theory (DFT) calculations with the correction of Hubbard $U$ parameter $(\mathrm{DFT}+U)$ were employed $[24,25]$. We chose $U=7 \mathrm{eV}$ [26] to improve the prediction of the computation. And a $500 \mathrm{eV}$ plane-wave energy cutoff was used to expand the electronic wave functions. The $7 \times 7 \times 7$ Monkhorst-Pack grid was used for the sampling of the Brillouin zone during geometrical optimization. A supercell of $2 \times 2 \times 2$ without any vacancy and with different oxygen vacancies was built to simulate the magnetic properties of $\mathrm{CeO}_{2}$. All the atomic positions and lattice parameters were relaxed and optimized until the convergence of force reaches $0.01 \mathrm{eV} \AA^{-1}$.

\section{Results and Discussion}

A typical XRD pattern of the sample is shown in Fig. 1a. All the peaks can be indexed to the cubic phase (Fm $3 \mathrm{~m}$, JCPDS 34-0394) of $\mathrm{CeO}_{2}$ without any impurity peaks. FESEM images of the synthesized $\mathrm{CeO}_{2}$ sample, as shown in Fig. 1b, c, demonstrate that the particles are quite uniform and have regular cube-like morphology with a size of $200 \mathrm{~nm}$. TEM image in Fig. 1d also shows clear cubic shape of $\mathrm{CeO}_{2}$ particle. The inset is the high-resolution TEM image of the selected area, which has lattice planes (100) with $d$-spacing of $0.27 \mathrm{~nm}$.

Figure $2 \mathrm{a}, \mathrm{b}$ show UV-Vis reflectance spectra and Kubelka-Munk functions of the $\mathrm{CeO}_{2}$ nanocubes without and with UV irradiation. Extrapolating the linear part of Kubelka-Munk function, which is actually the ratio of the absorption and scattering factors from the optical diffuse 

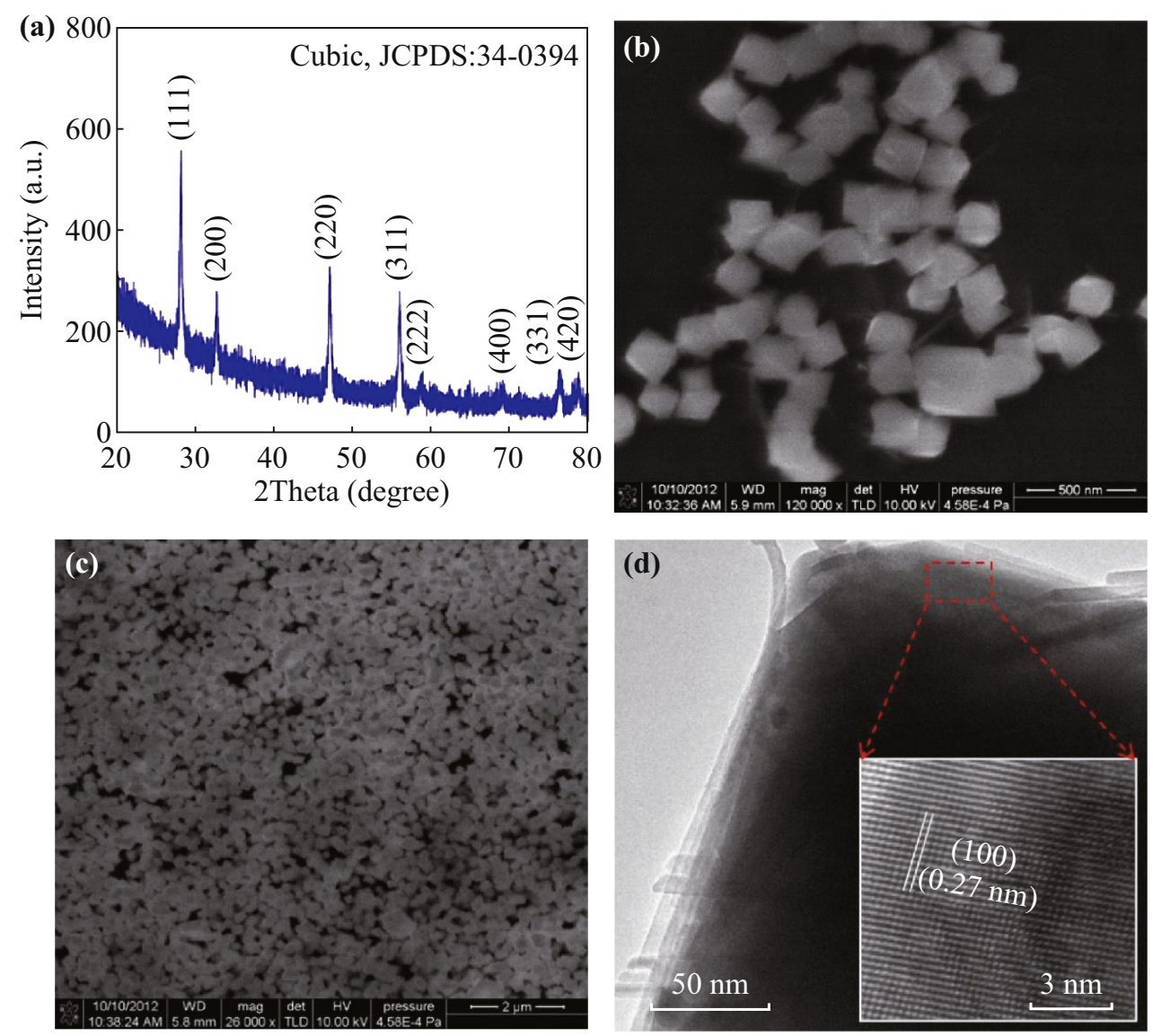

Fig. 1 a XRD, b, c FESEM images, and d TEM image of the $\mathrm{CeO}_{2}$ sample

reflectance spectrum [27], the energy gap of the $\mathrm{CeO}_{2}$ can be evaluated as 3.13 and $3.04 \mathrm{eV}$ for the $\mathrm{CeO}_{2}$ sample without and with UV irradiation, respectively (Fig. 2a, b) $[28,29]$. The reduced band gap can be attributed to the defect energy level, as some previous studies have reported that oxygen deficiencies create additional energy level within the forbidden energy gap [30,31].

Since the intensity of $\mathrm{Ce} 4 \mathrm{p}$ and $\mathrm{Ce} 4 \mathrm{~d}$ is much lower compared to that of Ce $3 \mathrm{~d}$, we choose Ce $3 \mathrm{~d}$ signal in XPS results to analyze further. Figure 3 a shows the photoelectron spectrum of Ce $3 \mathrm{~d}$ core level before UV irradiation, among which $\mathrm{V}$ and $\mathrm{U}$ are for $\mathrm{Ce}^{3+}$ and $\mathrm{Ce}^{4+}$ states, respectively. The binding energy peaks labeled as $\mathrm{V} 1$ and $\mathrm{V} 2$ are located at $883.26 \mathrm{eV}$ and $902.81 \mathrm{eV}$, indicating the $\mathrm{Ce}^{3+} 3 \mathrm{~d}_{5 / 2}$ and $\mathrm{Ce}^{3+} 3 \mathrm{~d}_{3 / 2}$. The peaks (U1, U2 U3, U4, U5, and U6) of $\mathrm{Ce}^{4+} 3 \mathrm{~d}_{5 / 2}$ and $\mathrm{Ce}^{4+} 3 \mathrm{~d}_{3 / 2}$ are respectively shown at binding energy of $881.48,888.34,897.73,899.85$, 907.05, and $916.34 \mathrm{eV}$ [32-34]. Compared with the sample after UV irradiation in Fig. 3b, it can be found that positions of $\mathrm{Ce} 3 \mathrm{~d}$ peaks are nearly unchanged. However, the content of elements changes and the intensity ratio of $\mathrm{Ce}^{3+}$ to $\mathrm{Ce}^{4+}$ increases from 0.260 to 0.332 . This may be ascribed to the increase of $\mathrm{Ce}^{3+}$ ions after UV irradiation.
Figure $3 \mathrm{c}$ shows the $\mathrm{O} 2 \mathrm{p}$ core level of the sample before UV irradiation, labeled as $\mathrm{O} 1, \mathrm{O} 2$, and $\mathrm{O} 3$, which indicates the valence of $\mathrm{O}$ is -2 . The levels of $\mathrm{O} 2 \mathrm{p}$ peaks at 528.83 and $530.86 \mathrm{eV}$ are ascribed to $\mathrm{O}^{2-}$ ions which are related to $\mathrm{Ce}^{4+}$ and $\mathrm{Ce}^{3+}$ ions [33], while the peak of $532.47 \mathrm{eV}$ is attributed to the oxygen absorbed onto the surface of the samples [35, 36]. Same with Ce $3 d$, the positions of O $2 p$ peaks after UV irradiation do not shift while the relative intensity of these peaks changes (Fig. 3d), which indicates the change of element quantity. In calculation results, the intensity ratio of $\mathrm{O} 2$ to $\mathrm{O} 1$ increases from 1.183 to 1.291 , demonstrating that the $\mathrm{O}^{2-}$ ions related to $\mathrm{Ce}^{3+}$ increase, which confirms the increase in oxygen vacancies. All the curve-fitting processes in Fig. 3 use a mixed GaussianLorentzian simulation method with the same full width at half-maximum.

Figure 4 shows the hysteresis loops of sample from $-15,000$ Oe to $+15,000$ Oe before and after UV irradiation. The saturation magnetization of the sample before UV irradiation is $3.18 \times 10^{-3} \mathrm{emu} \mathrm{g}^{-1}$, while it was greatly enhanced to $1.89 \times 10^{-2} \mathrm{emu} \mathrm{g}^{-1}$ after UV irradiation. This may relate to the increase of oxygen vacancies caused by UV irradiation. 

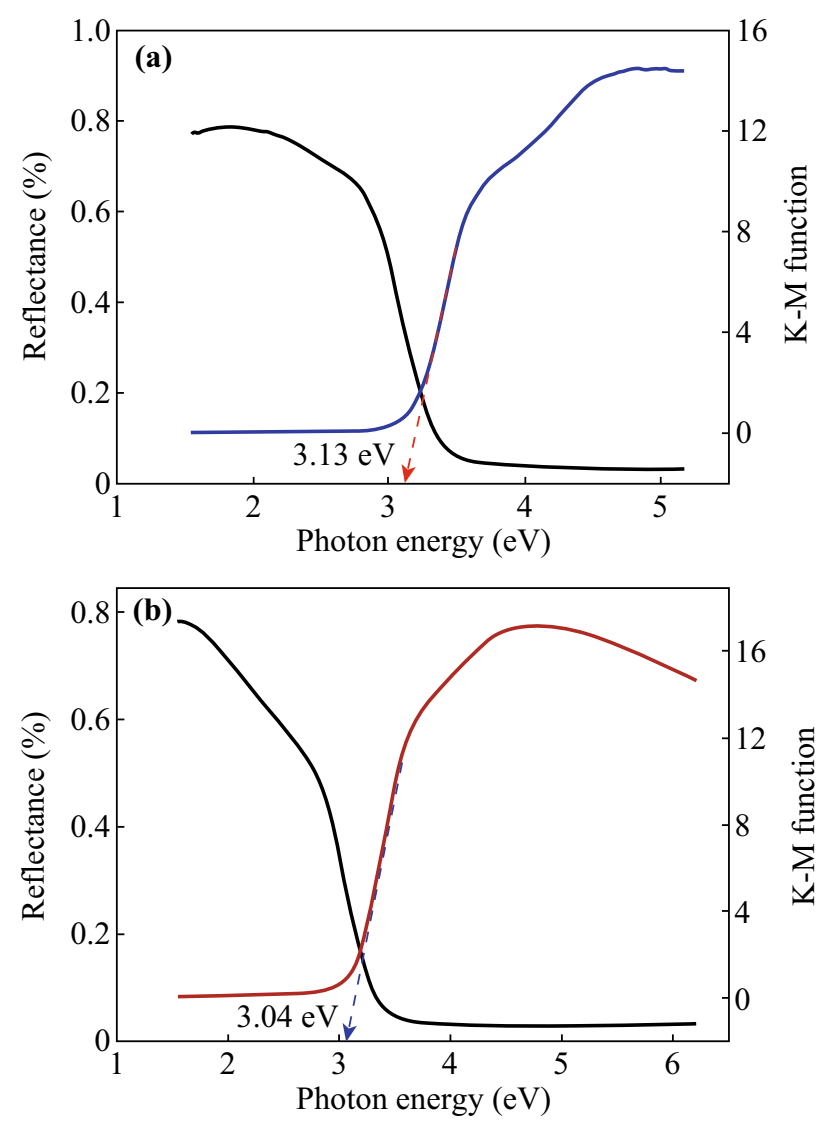

Fig. 2 UV-Visible reflectance spectrum and Kubelka-Munk function of the $\mathrm{CeO}_{2}$ sample before (a) and after (b) UV irradiation
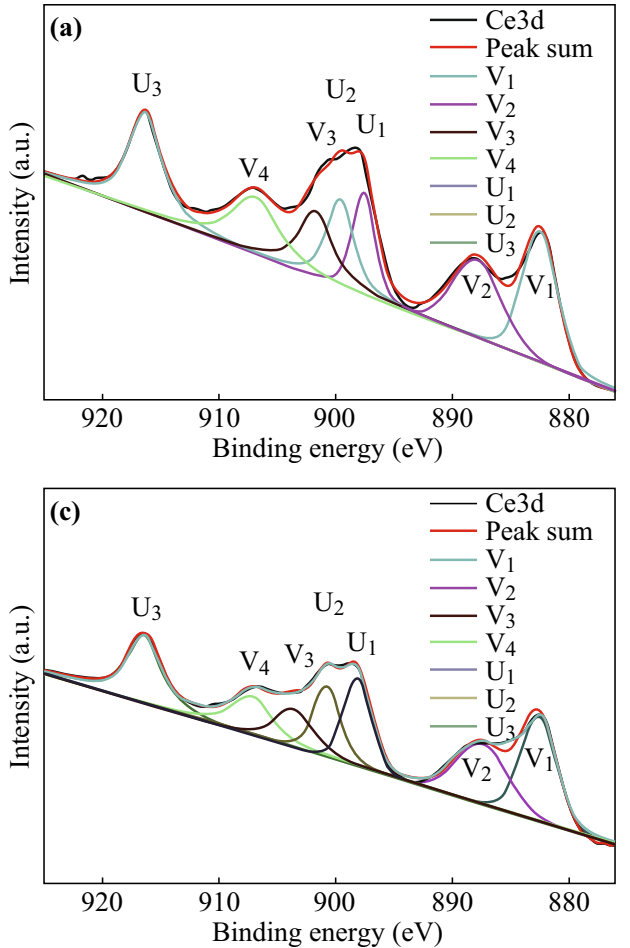

From the above discussion, we have demonstrated that UV irradiation can generate oxygen vacancies, and oxygen vacancies will induce the enhancement of magnetism. In order to understand the mechanism, electron DOS, projected density of states (PDOS), and the magnetism of the structure were calculated using the first-principle calculation in the VASP. In all calculations, the projector augmented wave method (PAW) [37] with the frozen-core approximation was used for the ion-electron interactions. Exchange correlation interactions were described by the generalized gradient approximation (GGA) [38].

The DOSs and PDOSs of $\mathrm{CeO}_{2}$ without and with one oxygen vacancy and two oxygen vacancies were calculated and the results are shown in Fig. 5. One can see the perfect structure of tetrahedral $\mathrm{CeO}_{2}$ supercell with 32 cerium atoms and 64 oxygen atoms. Each oxygen atom is surrounded by four cerium atoms with the same distance. To simplify the atom orbitals, the $\mathrm{f}$ orbitals of $\mathrm{Ce} 4 \mathrm{f}$ are marked as $4 \mathrm{f}-3 \sim 4 \mathrm{f} 3$ and the $\mathrm{p}$ orbitals of $\mathrm{O} 2 \mathrm{p}$ are marked as $\mathrm{P}_{\mathrm{x}} \sim \mathrm{P}_{\mathrm{z}}$ as shown in Fig. 5b. From the total DOS of $\mathrm{CeO}_{2}$, all the electron spin-up and spin-down states are symmetric and show no magnetism in the perfect $\mathrm{CeO}_{2}$ cell. Comparing the $\mathrm{Ce} 4 \mathrm{f}$ state with $\mathrm{O} 2 \mathrm{p}$, we have observed that $\mathrm{O} 2 \mathrm{p}$ contributes more to the total DOS at the top valence band than the $\mathrm{Ce} 4 \mathrm{f}$. While at the bottom conduction band the majority was formed by $\mathrm{Ce} 4 \mathrm{f}$. The integrative action of $\mathrm{Ce} 4 \mathrm{f}$ and $\mathrm{O} 2 \mathrm{p}$ makes the $\mathrm{CeO}_{2}$ nonmagnetic. When one oxygen is removed, an oxygen
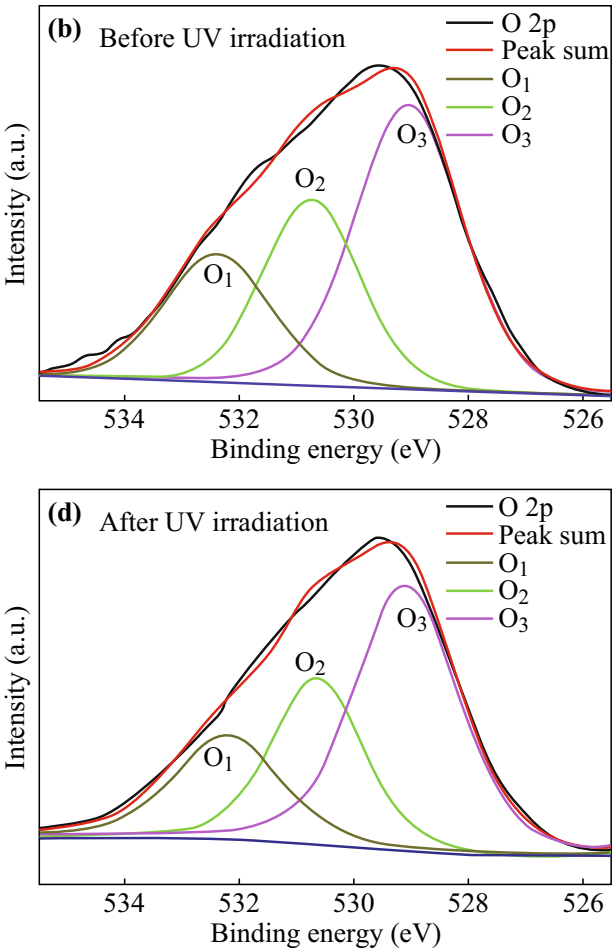

Fig. 3 a Ce $3 \mathrm{~d}$ core level, and b O $2 p$ core level for the $\mathrm{CeO}_{2}$ sample before UV irradiation. c Ce $3 \mathrm{~d}$ core level, and d O $2 p$ core level for the $\mathrm{CeO}_{2}$ sample after UV irradiation 
vacancy is generated, as shown in Fig. 5c. Several PDOSs of $\mathrm{Ce} 4 \mathrm{f}$ are symmetric except $\mathrm{f}-3, \mathrm{f}-2$, and $\mathrm{f} 3$, which generate most of the magnetism of $\mathrm{CeO}_{2}$ with one vacancy. From the PDOSs of $\mathrm{O} 2 \mathrm{p}$, the $\mathrm{P}_{\mathrm{x}}, \mathrm{P}_{\mathrm{y}}$, and $\mathrm{P}_{\mathrm{z}}$ states are symmetric at the top valence band constituting most of the valence band of $\mathrm{CeO}_{2}$, as can be seen from Fig. $5 \mathrm{~d}$. Meanwhile, at the bottom conduction band, $\mathrm{O} 2 \mathrm{p}$ exhibits slight asymmetry which contributes to the formation of the conduction band of $\mathrm{CeO}_{2}$. With one oxygen vacancy, the calculation of magnetism of the sample is about $1.213 \mu \mathrm{B}$. After another oxygen atom is removed, two oxygen vacancies are formed. Herein, we choose two cases to analyze the magnetism as depicted in Fig. 5e, g. Comparing the DOSs and PDOSs of Ce $4 \mathrm{f}$ and $\mathrm{O} 2 \mathrm{p}$ in Fig. 5f, h, it is not difficult for us to discover that all their electron spin-

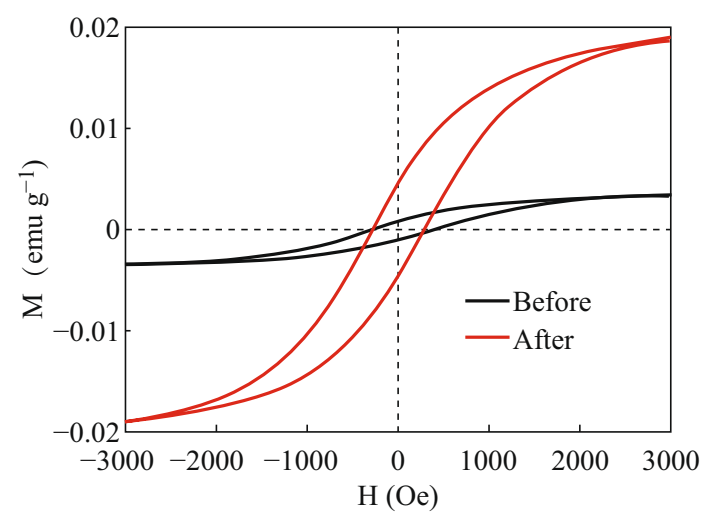

Fig. 4 Hysteresis loops of the $\mathrm{CeO}_{2}$ sample before and after UV irradiation measured at room temperature up and spin-down in $\mathrm{f}$ orbitals of $\mathrm{Ce} 4 \mathrm{f}$ are asymmetric, generating most of the magnetism of $\mathrm{CeO}_{2}$. Same as the situation in Fig. 5b, the electron spin-up and spin-down of $\mathrm{O} 2 \mathrm{p}$ are symmetric at the top valence band and reveal a bit of asymmetry at the bottom conduction band. Since the asymmetry of PDOSs in $\mathrm{f}$ orbitals in Fig. $5 \mathrm{~h}$ is higher than that of PDOSs in $\mathrm{f}$ orbitals in Fig. 5f, the magnetizaiton of the sample in Fig. 5h is larger than that in Fig. $5 \mathrm{f}$ in accordance with the values in the figure $\left(M_{2}=1.912 \mu \mathrm{B}\right.$, $\left.M_{2^{\prime}}=1.968 \mu \mathrm{B}\right)$. Moreover, the magnetization of the sample with two oxygen vacancies is obviously larger than that with one oxygen vacancy, which well matches our demonstration that the enhancement of the magnetism of $\mathrm{CeO}_{2}$ after UV irradiation is due to the generation of oxygen vacancies. Since the oxygen vacancy exhibits positive electricity, it attracts nearest oxygen atoms. The blue arrows in Fig. 5c, e, g demonstrate the displacement of the neighboring oxygen atoms. All the structures of $\mathrm{CeO}_{2}$ without and with oxygen vacancies are optimized in the calculations of the DOSs, PDOSs, and magnetism.

Figure 6a shows the schematic of superexchange interaction among $1 \mathrm{Ce}, 2 \mathrm{Ce}$, and $\mathrm{O}$ atoms. The two $\mathrm{Ce}$ atoms are respectively reduced by two electrons from the oxygen vacancy, forming a $\mathrm{Ce}-\mathrm{O}-\mathrm{Ce}$ bond together with the neighboring $\mathrm{O}$ atom, which favors a $90^{\circ}$ superexchange interaction, leading to magnetism [17, 39]. Figure $6 \mathrm{~b}-\mathrm{f}$ display the process of superexchange interaction of $\mathrm{Ce}-\mathrm{O}-$ $\mathrm{Ce}$. First, one electron on one $\mathrm{O} 2 \mathrm{p}$ orbital is excited by UV irradiation to one $4 \mathrm{f}$ orbital of $1 \mathrm{Ce}$ atom (Fig. 6c), and its spin direction is parallel to the electron on Ce $4 \mathrm{f}$ orbital based on Hund's rule [40] (Fig. 6d). Since the $\mathrm{Ce}-\mathrm{O}-\mathrm{Ce}$ bond angle is close to $90^{\circ}$, the electron with the same spin

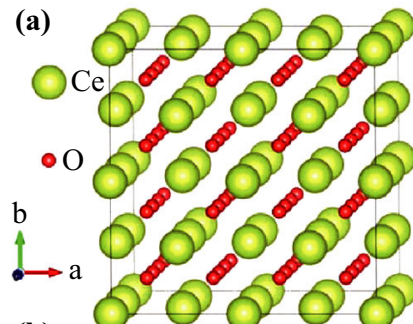

(b)

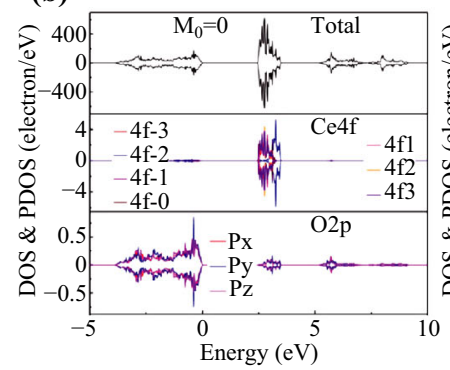

(c)

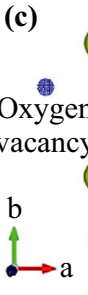

(d)

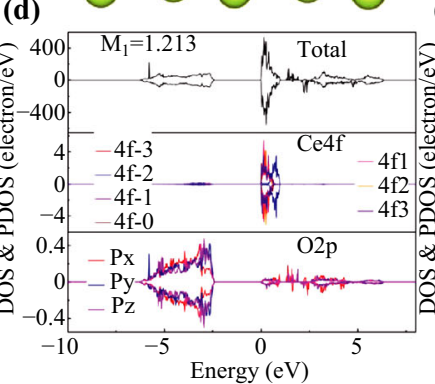

(e)

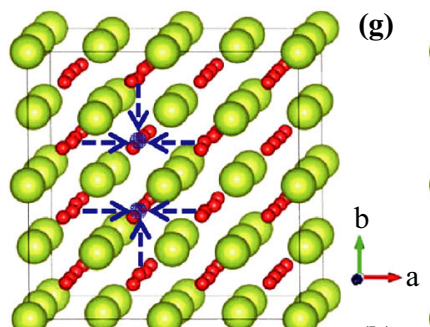

(f) (h)

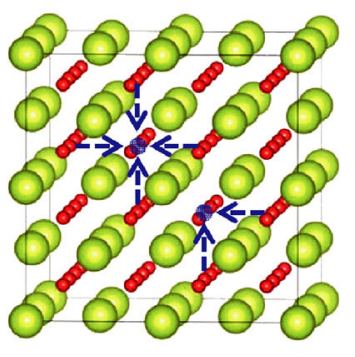

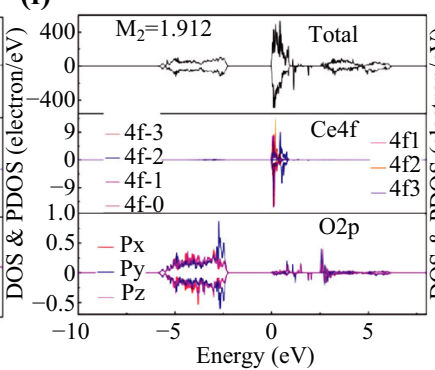

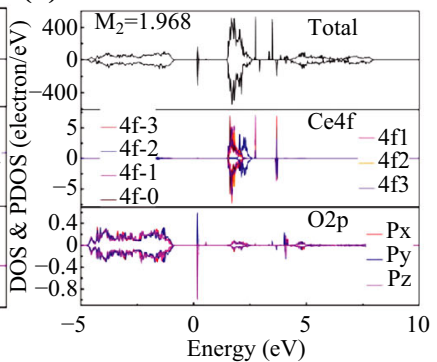

Fig. 5 The schematic of $\mathrm{CeO}_{2}$ supercell without any oxygen vacancy (a), with one oxygen vacancy (c), two oxygen vacancies (e, g), and their corresponding DOSs and PDOSs (b, $\mathbf{d}, \mathbf{f}$, and $\mathbf{h}$ ) 

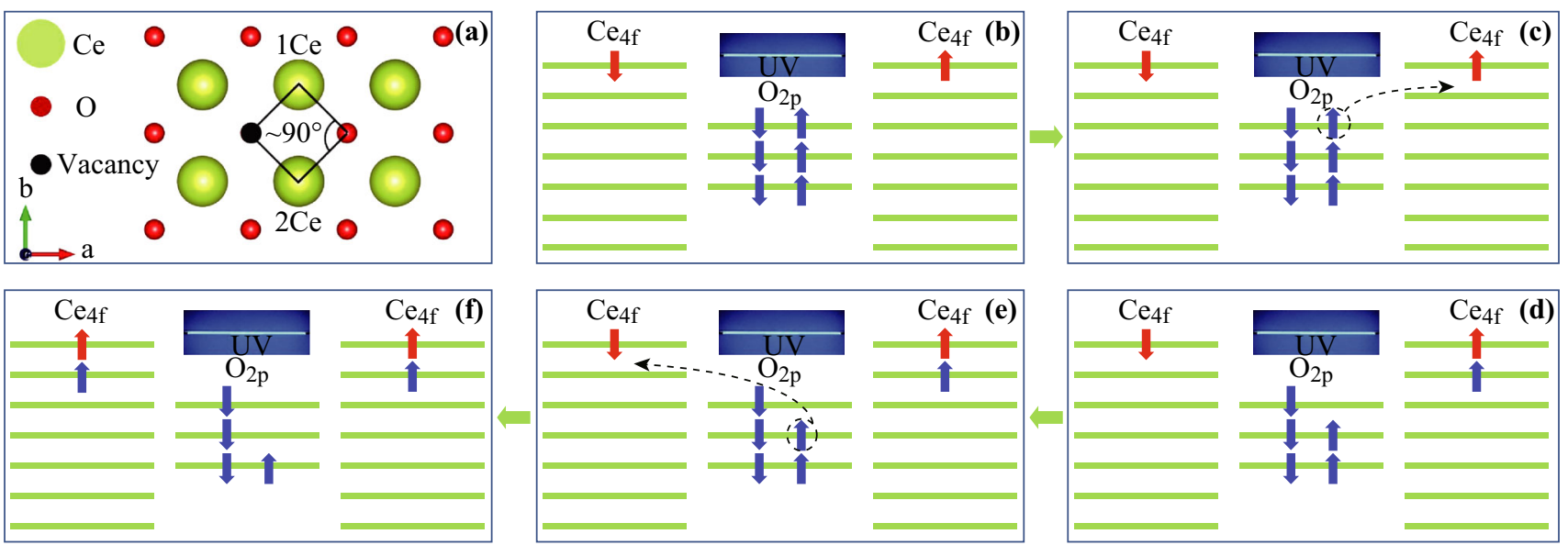

Fig. 6 The schematic of superexchange interaction among $1 \mathrm{Ce}, 2 \mathrm{Ce}$, and $\mathrm{O}(\mathbf{a})$, and the process of superexchange interaction of Ce-O-Ce (b-f)

direction on another $\mathrm{O} 2 \mathrm{p}$ orbital is exchanged with $4 \mathrm{f}$ orbital of $2 \mathrm{Ce}$ atom (Fig. 6e), which makes the spin direction of the electron on $2 \mathrm{Ce} 4 \mathrm{f}$ orbital parallel to it (Fig. 6f). Therefore, the magnetic moments of these two Ce atoms have the same direction, generating ferromagnetic coupling. With the increase in oxygen vacancies, the tetrahedral structure of the sample is further destroyed, making the $\mathrm{Ce}$ atoms close to the oxygen vacancies, which increases the total magnetic momentum. Back to Fig. 5, as UV irradiation produces more oxygen vacancies in the structure of $\mathrm{CeO}_{2}$, the electron spin on $\mathrm{Ce} 4 \mathrm{f}$ orbitals becomes more complicated, making the PDOSs of Ce $4 \mathrm{f}$ more asymmetric. Thus UV irradiation can enhance the magnetism by producing oxygen vacancies.

\section{Conclusions}

In this work, uniform $\mathrm{CeO}_{2}$ nanocubes were synthesized and oxygen vacancy can be increased and therefore the magnetism was enhanced by UV irradiation. The firstprinciple calculation was used to explain the magnetism enhancement mechanism. According to the calculation results, the increase of oxygen vacancy which confirmed by XPS measurements will enhance the DOSs of Ce4f orbital, and therefore superexchange interaction of $\mathrm{Ce}-\mathrm{O}-\mathrm{Ce}$ generates magnetism. This work demonstrates an effective route to produce diluted magnetism for $\mathrm{CeO}_{2}$ nanocubes and offers a better understanding of room-temperature magnetism in $\mathrm{CeO}_{2}$ nanocrystals.

Acknowledgments This work is supported by the National High Technology Research and Development Program (863 program) of China (2015AA034801), NSFC (11204388, 51402112), the Fundamental Research Funds for the Central Universities (CQDXWL-2014001 and CQDXWL-2013-012), and the large-scale equipment sharing fund of Chongqing University.
Open Access This article is distributed under the terms of the Creative Commons Attribution 4.0 International License (http://crea tivecommons.org/licenses/by/4.0/), which permits unrestricted use, distribution, and reproduction in any medium, provided you give appropriate credit to the original author(s) and the source, provide a link to the Creative Commons license, and indicate if changes were made.

\section{References}

1. X.L. Wang, S.Z. Yi, E.W. Liang, Y.Y. Wu, Z.X. Fang, Study on preparation of polishing powder for LCD. Adv. Mater. Res. 785-786, 480-483 (2013). doi:10.4028/www.scientific.net/AMR. 785-786.480

2. Y. Chen, Z.N. Li, N.M. Miao, Polymethylmethacrylate (PMMA)/ $\mathrm{CeO}_{2}$ hybrid particles for enhanced chemical mechanical polishing performance. Tribol. Int. 82, 211-217 (2015). doi:10.1016/ j.triboint.2014.10.013

3. M. Ozawa, Role of cerium-zirconium mixed oxides as catalysts for car pollution: a short review. J. Alloy. Compd. 275, 886-890 (1998). doi:10.1016/S0925-8388(98)00477-0

4. S.Y. Christou, S. Garacia-Rodriguez, J.L.G. Fierro, A.M. Efstathiou, Deactivation of $\mathrm{Pd} / \mathrm{Ce}_{0.5} \mathrm{Zr}_{0.5} \mathrm{O}_{2}$ model three-way catalyst by $\mathrm{P}, \mathrm{Ca}$ and $\mathrm{Zn}$ deposition. Appl. Catal. B: Environ. 111, 233-245 (2012). doi:10.1016/j.apcatb.2011.10.004

5. Z.X. Yang, X.H. Yu, Z.S. Lu, S.F. Li, K. Hermansson, Oxygen vacancy pairs on $\mathrm{CeO}_{2}(110)$ : a DFT+U study. Phys. Lett. A 373(31), 2786-2792 (2009). doi:10.1016/j.physleta.2009.05.055

6. K.C. Anjaneya, G.P. Nayaka, J. Manjanna, G. Govindaraj, K.N. Ganesha, Preparation and characterization of $\mathrm{Ce}_{1-\mathrm{x}} \mathrm{Gd}_{\mathrm{x}} \mathrm{O}_{2}$-delta $(\mathrm{x}=0.1-0.3)$ as solid electrolyte for intermediate temperature SOFC. J. Alloy. Compd. 578, 53-59 (2013). doi:10.1016/j.jall com.2013.05.010

7. E. Bekyarova, P. Fornasiero, J. Kaspar, M. Graziani, CO oxidation on $\mathrm{Pd} / \mathrm{CeO}_{2}-\mathrm{ZrO}_{2}$ catalysts. Catal. Today $45(1-4), 179-183$ (1998). doi:10.1016/S0920-5861(98)00212-0

8. H. Yahiro, Y. Baba, K. Eguchi, H. Arai, High-temperature fuelcell with ceria-yttria solid electrolyte. J. Electrochem. Soc. 135(8), 2077-2080 (1988). doi:10.1149/1.2096212

9. M.F. Al-Kuhaili, S.M.A. Durrani, I.A. Bakhtiari, Carbon monoxide gas-sensing of $\mathrm{CeO}_{2}-\mathrm{ZnO}$ thin films. Appl. Surf. Sci. 255(5), 3033-3039 (2008). doi:10.1016/j.apsusc.2008.08.058

10. B. Choudhury, A. Choudhury, $\mathrm{Ce}^{3+}$ and oxygen vacancy mediated tuning of structural and optical properties of $\mathrm{CeO}_{2}$ 
nanoparticles. Mater. Chem. Phys. 131(3), 666-671 (2012). doi:10.1016/j.matchemphys.2011.10.032

11. Y. Taga, Recent progress in coating technology for surface modification of automotive glass. J. Non-Cryst. Solids 218, 335-341 (1997). doi:10.1016/S0022-3093(97)00281-0

12. S. Zec, S. Boskovic, B. Kaluderovic, Z. Bogdanov, N. Popovic, Chemical reduction of nanocrystalline $\mathrm{CeO}_{2}$. Ceram. Int. 35(1), 195-198 (2009). doi:10.1016/j.ceramint.2007.10.031

13. L. Zhang, H. Jiang, M. Selke, X.M. Wang, Selective cytotoxicity effect of cerium oxide nanoparticles under UV irradiation. J. Biomed. Nanotechnol. 10(2), 278-286 (2014). doi:10.1166/jbn. 2014.1790

14. R. Si, M. Flytzani-Stephanopoulos, Shape and crystal-plane effects of nanoscale ceria on the activity of $\mathrm{Au}-\mathrm{CeO}_{2}$ catalysts for the water-gas shift reaction. Angew. Chem. Int. Edit. 47(15), 2884-2887 (2008). doi:10.1002/anie.200705828

15. N. Izu, S. Nishizaki, T. Itoh, M. Nishibori, W. Shin, I. Matsubara, Gas response, response time and selectivity of a resistive $\mathrm{CO}$ sensor based on two connected $\mathrm{CeO}_{2}$ thick films with various particles sizes. Sens. Actuators B 136(2), 364-370 (2009). doi:10. 1016/j.snb.2008.12.018

16. M. Nolan, Hybrid density functional theory description of oxygen vacancies in the $\mathrm{CeO}_{2}$ (110) and (100). Chem. Phys. Lett. 499(1-3), 126-130 (2010). doi:10.1016/j.cplett.2010.09.016

17. X.P. Han, J.C. Lee, H.I. Yoo, Oxygen-vacancy-induced ferromagnetism in $\mathrm{CeO}_{2}$ from first principles. Phys. Rev. B 79(10), 100403 (2009). doi:10.1103/PhysRevB.79.100403

18. S. Phokha, S. Pinitsoontorn, S. Maensiri, Structure and magnetic properties of monodisperse $\mathrm{Fe}^{3+}$-doped $\mathrm{CeO}_{2}$ nanospheres. Nano-Micro Lett. 5(4), 223-233 (2013). doi:10.5101/nml.v5i4. p223-233

19. M.S. Si, D.Q. Gao, D.Z. Yang, Y. Peng, Z.Y. Zhang, D.S. Xue, Y.S. Liu, X.H. Deng, G.P. Zhang, Intrinsic ferromagnetism in hexagonal boron nitride nanosheets. J. Chem. Phys. 140, 204701 (2014). doi:10.1063/1.4879055

20. C. Klein, R. Ramchal, A.K. Schmid, M. Farle, Controlling the kinetic order of spin-reorientation transitions in $\mathrm{Ni} / \mathrm{Cu}(100)$ films by tuning the substrate step structure. Phys. Rev. B 75, 193405 (2007). doi:10.1103/PhysRevB.75.193405

21. S. Qin, D. Liu, Z. Zuo, Y. Sang, H. Liu, UV-irradiation-enhanced ferromagnetism in $\mathrm{BaTiO}_{3}$. J. Phys. Chem. Lett. 1(1), 238-241 (2010). doi:10.1021/jz900131x

22. L. Feng, D.T. Hoang, C.K. Tsung, W.Y. Huang, S.H.Y. Lo, J.B. Wood, H. Wang, J.Y. Tang, P.D. Yang, Catalytic properties of Pt cluster-decorated $\mathrm{CeO}_{2}$ nanostructures. Nano Res. 4(1), 61-71 (2011). doi:10.1007/s12274-010-0042-4

23. G. Kresse, J. Furthmuller, Efficient iterative schemes for ab initio total-energy calculations using a plane-wave basis set. Phys. Rev. B 54(16), 11169-11186 (1996). doi:10.1103/PhysRevB.54.11169

24. D.A. Andersson, S.I. Simak, N.V. Skorodumova, I.A. Abrikosov, B. Johansson, Theoretical study of $\mathrm{CeO}_{2}$ doped with tetravalent ions. Phys. Rev. B 76(17), 174119 (2007). doi:10.1103/Phys RevB.76.174119

25. M. Nolan, S.C. Parker, G.W. Watson, The electron structure of oxygen vacancy defects at the low index surfaces of ceria. Surf. Sci. 595(1-3), 223-232 (2005). doi:10.1016/j.susc.2005.08.015
26. C. Loschen, J. Carrasco, K.M. Neyman, F. Illas, First-principles $\mathrm{LDA}+\mathrm{U}$ and GGA $+\mathrm{U}$ study of cerium oxides: dependence on the effective U parameter. Phys. Rev. B 75, 035115 (2007). doi:10.1103/PhysRevB.75.035115

27. J. Li, Z. Chen, X.X. Wang, D.M. Proderpio, A novel two-dimensional mercury antimony telluride: low temperature synthesis and characterization of $\mathrm{RbHgSbTe}_{3}$. J. Alloys Compd. 262, 28-33 (1997). doi:10.1016/S0925-8388(97)00324-1

28. J. Xu, C.G. Hu, Y. Xi, C. Peng, B.Y. Wan, X.S. He, Synthesis, photoluminescence and magnetic properties of barium vanadate nanoflowers. Mater. Res. Bull. 46(6), 946-950 (2011). doi:10. 1016/j.materresbull.2011.02.023

29. X.A. Huang, D. Kocaefe, Y. Kocaefe, Y. Boluk, A. Pichette, A spectrocolorimetric and chemical study on color modification of heat-treated wood during artificial weathering. Appl. Surf. Sci. 258(14), 5360-5369 (2012). doi:10.1016/j.apsusc.2012.02.005

30. D.C. Cronemeyer, Infrared absorption of reduced rutile $\mathrm{TiO}_{2}$ single crystals. Phys. Rev. 113(5), 1222-1226 (1959). doi:10. 1103/PhysRev.113.1222

31. D.L. Guo, C.G. Hu, Y. Xi, UV-irradiation-enhanced ferromagnetism of barium vanadate $\left(\mathrm{Ba}_{3} \mathrm{~V}_{2} \mathrm{O}_{8}\right)$ nanoflowers. J. Alloy. Compd. 550, 389-394 (2013). doi:10.1016/j.jallcom.2012.10.152

32. X.H. Lu, X. Huang, S.L. Xie, D.Z. Zheng, Z.Q. Liu, C.L. Liang, Y.X. Tong, Facile electrochemical synthesis of single crystalline $\mathrm{CeO}_{2}$ octahedrons and their optical properties. Langmuir 26(10), 7569-7573 (2010). doi:10.1021/la904882t

33. A. Arumugam, C. Karthikeyan, A.S.H. Hameed, K. Gopinath, S. Gowri, V. Karthika, Synthesis of cerium oxide nanoparticles using Gloriosa superba L. leaf extract and their structural, optical and antibacterial properties. Mater. Sci. Eng. C 49, 408-415 (2015). doi:10.1016/j.msec.2015.01.042

34. L.N. Wang, F.M. Meng, Oxygen vacancy and $\mathrm{Ce}^{3+}$ ion dependent magnetism of monocrystal $\mathrm{CeO}_{2}$ nanopoles synthesized by a facile hydrothermal method. Mater. Res. Bull. 48(9), 3492-3498 (2013). doi:10.1016/j.materresbull.2013.05.036

35. Z. Zhang, C. Hu, M. Hashim, P. Chen, Y. Xiong, C. Zhang, Synthesis and magnetic properties of $\mathrm{FeMoO}_{4}$ nanorods. Mater. Sci. Eng. B 176(9), 756-761 (2011). doi:10.1016/j.mseb.2011.02. 018

36. X.Y. Wu, J. Du, H.B. Li, M.F. Zhang, B.J. Fan, Y.C. Zhu, Y.T. Qian, Aqueous mineralization process to synthesize uniform shuttle-like $\mathrm{BaMoO}_{4}$ microcrystals at room temperature. J. Solid State Chem. 180(11), 3288-3295 (2007). doi:10.1016/j.jssc.2007. 07.010

37. G. Kresse, D. Joubert, From ultrasoft pseudopotential to the projector augmented-wave method. Phys. Rev. B 59(3), 1758-1775 (1999). doi:10.1103/PhysRevB.59.1758

38. J.P. Perdew, K. Burke, M. Ernzerhof, Generalized gradient approximation made simple. Phys. Rev. Lett. 77(18), 3865-3868 (1996). doi:10.1103/PhysRevLett.77.386

39. J.B. Goodenough, Magnetism and the Chemical Bond (Interscience, New York, 1963)

40. S. Yamanaka, K. Koizumi, Y. Kitagawa, T. Kawakami, M. Okumura, K. Yamaguchi, Chemical bonding, less screening, and Hund's rule revisted. Int. J. Quantum Chem. 105(6), 687-700 (2005). doi:10.1002/qua.20784 